Notre Dame Journal of Formal Logic

Volume 42, Number 4, 2001

\title{
Anshakov-Rychkov Algebras
}

\author{
Olga Ambas
}

\begin{abstract}
The aim of this paper is to show that the calculi described by Anshakov and Rychkov are algebraizable in the sense of Blok and Pigozzi. As a consequence, a proof of the strong completeness of these calculi is obtained.
\end{abstract}

\section{Introduction}

The aim of this paper is to give an algebraic treatment of the finite-valued calculi given by Anshakov and Rychkov [2]. These calculi are very close to the ones considered by Rosser and Turquette [8]. Particular cases are classical logic and Moisil $n$-valued logics.

Let $n \geq 2$ and $\mathbb{F}=\left\{J_{0}, J_{1}, \ldots, J_{n-1}\right\} \cup\{\neg, \vee, \wedge, \supset\} \cup \mathbb{G}$ be a finite set of finitary operations such that $\neg, J_{i}, i=0, \ldots, n-1$ are unary operations and $\vee, \wedge, \supset$ are binary operations. Anshakov-Rychkov algebras are algebras of type $\mathbb{E}$ such that they satisfy natural axioms that correspond to the axioms of the propositional calculus. We are going to prove that these algebras are subdirect products of subalgebras of special algebras $\mathbf{M}_{n}=\left\langle M_{n}, \mathbb{F}\right\rangle$, where $M_{n}=\left\{0, \frac{1}{n-1}, \ldots, \frac{n-2}{n-1}, 1\right\}$. This fact is used to proof the strong completeness of these calculi with respect to the semantics given by the matrices $\mathbf{M}_{n}$. More precisely, we are going to prove that the AnshakovRychkov calculi are algebraizable in the sense of Blok and Pigozzi [3] with equivalent algebraic semantic $\mathcal{M}$, where $\mathcal{M}$ is a quasi variety which depends naturally on $\mathbf{M}_{n}$.

\section{Anshakov-Rychkov Algebras}

In what follows, $n$ will always denote an integer $\geq 2$. Let $\mathbb{F}=\left\{J_{0}, J_{1}, \ldots, J_{n-1}\right\} \cup$ $\{\neg, \vee, \wedge, \supset\} \cup \mathbb{G}$ be a finite set of finitary operations such that $\neg, J_{i}, i=0, \ldots, n-1$ are unary operations and $\vee, \wedge, \supset$ are binary operations. The set $\mathbb{G}$ may be empty. We denote by $\mathcal{L}_{\mathbb{F}}$ the language of algebras given by $\mathbb{F}$.

Received January 4, 2001; accepted July 5, 2003; printed August 29, 2003

2001 Mathematics Subject Classification: Primary, 03B50; Secondary, 03G25

Keywords: many-valued logic, algebraizable, completeness, quasi variety (c) 2003 University of Notre Dame 
Definition 2.1 An n-matrix of type $\mathbb{F}$ is an algebra of language $\mathcal{L}_{\mathbb{F}}$ such that its universe $M_{n}$ is the set of rational fractions $\left\{0, \frac{1}{n-1}, \ldots, \frac{n-2}{n-1}, 1\right\}$ and the operations in $\mathbb{F}$ satisfy the following conditions, for every $x, y, z$ in $M_{n}$ :

$\mathrm{C}_{J_{i}} \quad J_{i}\left(\frac{j}{n-1}\right)= \begin{cases}1 & \text { if } j=i \\ 0 & \text { if } j \neq i\end{cases}$

$\mathrm{C}_{\{0,1\}}$ The set $\{0,1\}$ is closed under the operations $\neg, \vee, \wedge, \supset$, and

$$
\langle\{0,1\}, \neg, \vee, \wedge, \supset, 0,1\rangle
$$

is a Boolean algebra with the usual meaning of the operation $\neg, \vee, \wedge, \supset$;

$\mathrm{C}_{\wedge} \quad x \wedge y=1$ iff $x=y=1$;

$\mathrm{C}_{\vee} \quad x \vee y=1$ iff $x=1$ or $y=1$;

$\mathrm{C}_{\supset 1} \quad x \supset(y \supset x)=1$;

$\mathrm{C}_{\supset 2} \quad(x \supset(y \supset z)) \supset((x \supset y) \supset(x \supset z))=1$;

$\mathrm{C}_{\supset 3} \quad 1 \supset x=1$ iff $x=1$.

Observe that we are considering no order relation on the set $\left\{0, \frac{1}{n-1}, \ldots, \frac{n-2}{n-1}, 1\right\}$ besides $0<1$.

Given an algebra $\mathbf{A}=\langle A, \mathbb{F}\rangle$ of language $\mathcal{L}_{\mathbb{F}}$, the elements of the set

$$
E(\mathbf{A})=\left\{J_{i}(a): a \in A, i=0, \ldots, n-1\right\}
$$

are called the exterior elements of $A$.

Definition 2.2 Let $\mathbf{M}$ be an $n$-matrix of type $\mathbb{F}$ ( $n$ an integer $\geq 2$ ). $\mathbf{A}$ is an $L_{n^{-}}$ algebra for $\mathbf{M}$ or an Anshakov-Rychkov algebra of $\mathbb{F}$ provided that the following conditions hold.

Axiom 2.3 ( $n$-valuedness)

$n-\operatorname{Val}_{i} \quad J_{i}(a)=\bigwedge_{j \neq i} \neg J_{j}(a), i=0, \ldots, n-1$.

\section{Axiom 2.4 (connective J)}

$\begin{array}{ll}\mathrm{Cn} \mathrm{J}_{0} & J_{0}\left(J_{i}(a)\right)=\neg J_{i}(a), \\ \mathrm{Cn}-\mathrm{J}_{n-1} & J_{n-1}\left(J_{i}(a)\right)=J_{i}(a), \\ \mathrm{Cn}^{\mathrm{J}} \mathrm{J}_{i} & J_{i}\left(J_{j}(a)\right)=J_{n-1}(a) \wedge \neg J_{n-1}(a), i \notin\{0, n-1\} .\end{array}$

\section{Axiom 2.5 (closure)}

Cl-J $J_{*} \quad J_{i}(a) * J_{j}(b)=J_{n-1}\left(J_{i}(a) * J_{j}(b)\right)$, where $* \in\{\vee, \wedge, \supset\}$, $\mathrm{Cl}-\mathrm{J}_{\neg} \quad \neg J_{n-1}\left(J_{i}(a)\right)=J_{n-1}\left(\neg J_{i}(a)\right)$.

Observe that this axiom and $\mathrm{Cn}-\mathrm{J}_{0}$ imply that the set $E(\mathbf{A})$ is closed under the operations $\neg, \vee, \wedge$, and $\supset$.

\section{Axiom 2.6 (exterior elements)}

For any $x \in A, y \in A: J_{0}(x) \vee \neg J_{0}(x)=J_{0}(y) \vee \neg J_{0}(y)$.

Remark 2.7 We define $1:=J_{0}(x) \vee \neg J_{0}(x)$ and $0:=\neg 1$. Observe that from the previous axioms $\langle E(\mathbf{A}), \vee, \wedge, \supset, \neg, 0,1\rangle$ is a Boolean algebra.

\section{Axiom 2.8 (connective)}

Cn-G

$$
\begin{aligned}
& J_{i}\left(G\left(a_{1}, \ldots, a_{m}\right)\right)=\bigvee_{G\left(\frac{i_{1}}{n-1}, \ldots, \frac{i_{m}}{n-1}\right)=\frac{i}{n-1}} \bigwedge_{j=1}^{m} J_{i_{j}}\left(a_{j}\right), \text { for each } \\
& G \in \mathbb{F}-\left\{J_{i}, i=0, \ldots, n-1\right\} . \text { We define } \bigvee \varnothing=0 .
\end{aligned}
$$




\section{Axiom 2.9 (quasi identity)}

$$
\text { If } J_{i}(a)=J_{i}(b) \text { for all } i=0, \ldots, n-1 \text {, then } a=b \text {. }
$$

Remark 2.10 Observe that there is an axiom $\mathrm{Cn}$ for each connective in the set $\{\neg, \vee, \wedge, \supset\} \cup \mathbb{G}$. Hence, these axioms explicitly depend on the definitions of the operations in $\mathbb{G}$ on $\left\{0, \frac{1}{n-1}, \ldots, \frac{n-2}{n-1}, 1\right\}$ according to the matrix M. For each set $\mathbb{G}$, let $\mathcal{M}=\mathcal{M}(\mathbf{M})$ be the class of algebras of language $\mathcal{L}_{\mathbb{F}}$ axiomatized by Axiom $2.3-$ Axiom 2.9; that is, $\mathbf{A} \in \mathcal{M}$ if and only if $\mathbf{A}$ is an $L_{n}$-algebra for $\mathbf{M}$. Since these axioms are identities or quasi identities, it follows that $\mathcal{M}$ is a quasi variety.

Lemma 2.11 $\mathbf{M}$ is an $L_{n}$-algebra for the n-matrix $\mathbf{M}$ with $E(\mathbf{M})=\{0,1\}$, and it is the only possible structure of $L_{n}$-algebra for $\mathbf{M}$.

Proof The axioms are obviously satisfied. For instance, we check Axiom 2.8 (Cn-*), where $* \in \mathbb{F}=\{\neg, \vee, \wedge, \supset\} \cup \mathbb{G}$.

$J_{i}\left(*\left(a_{1}, a_{2}, \ldots, a_{k}\right)\right)=\bigvee_{*\left(\frac{i_{1}}{n-1}, \frac{i_{2}}{n-1}, \ldots, \frac{i_{k}}{n-1}\right)=\frac{i}{n-1}}\left(J_{i_{1}}\left(a_{1}\right) \wedge J_{i_{2}}\left(a_{2}\right) \wedge \cdots \wedge J_{i_{k}}\left(a_{k}\right)\right)$.

Since $a_{j} \in M_{n}, a_{j}=\frac{r_{j}}{n-1}, j=1, \ldots, k . J_{i}\left(*\left(a_{1}, a_{2}, \ldots, a_{k}\right)\right)=1$ if and only if $*\left(a_{1}, a_{2}, \ldots, a_{k}\right)=\frac{i}{n-1}$. On the other hand, from $\left(\mathrm{C}_{\vee}\right)$,

$$
\bigvee_{*\left(\frac{i_{1}}{n-1}, \frac{i_{2}}{n-1}, \ldots, \frac{i_{k}}{n-1}\right)=\frac{i}{n-1}}\left(J_{i_{1}}\left(\frac{r_{1}}{n-1}\right) \wedge J_{i_{2}}\left(\frac{r_{2}}{n-1}\right) \wedge \cdots \wedge J_{i_{k}}\left(\frac{r_{k}}{n-1}\right)\right)=1
$$

if and only if there are $i_{1}, i_{2}, \ldots, i_{k}$ such that $i_{j}=r_{j}, j=1, \ldots, k$ and $*\left(\frac{i_{1}}{n-1}, \frac{i_{2}}{n-1}, \ldots, \frac{i_{k}}{n-1}\right)=\frac{i}{n-1}$. Since $*\left(\frac{i_{1}}{n-1}, \frac{i_{2}}{n-1}, \ldots, \frac{i_{k}}{n-1}\right)=\frac{i}{n-1}$, this axiom is satisfied.

The connective axioms and Axiom 2.9 ensure that an $n$-matrix $\mathbf{M}$ admits only one structure of $L_{n}$-algebra.

Remark 2.12 Lemma 2.11 can be generalized as follows. If

$$
\mathbf{A}_{1}=\left\langle A, J_{0}, \ldots, J_{n-1}, \neg, \vee, \wedge, \supset, G_{1}, \ldots, G_{l}\right\rangle
$$

and

$$
\mathbf{A}_{2}=\left\langle A, J_{0}, \ldots, J_{n-1}, \bar{\neg}, \bar{\nabla}, \bar{\wedge}, \bar{\supset}, \overline{G_{1}}, \ldots, \overline{G_{l}}\right\rangle
$$

are $L_{n}$-algebras for $\mathbf{M}$, then $\mathbf{A}_{1}=\mathbf{A}_{2}$.

Lemma 2.13 Let $\mathbf{A}$ be an $L_{n}$-algebra for $\mathbf{M}$. The following properties hold for each $a \in A$.

1. $\bigvee_{i=0}^{n-1} J_{i}(a)=1$.

2. If $i \neq j$, then $J_{i}(a) \wedge J_{j}(a)=0$.

3. $J_{i}(a)=1$, then $J_{j}(a)=0$ for each $i \neq j$.

4. If $J_{n-1}(a)=1$, then $a=1$.

5. If $J_{0}(a)=1$, then $a=0$.

\section{Proof}

1. $\bigvee_{i=0}^{n-1} J_{i}(a)=J_{0}(a) \vee\left(\bigvee_{i=1}^{n-1} J_{i}(a)\right)=\left(\bigwedge_{j \neq 0} \neg J_{j}(a)\right) \vee\left(\bigvee_{i=1}^{n-1} J_{i}(a)\right)=$ $\neg\left(\bigvee_{j \neq 0} J_{j}(a)\right) \vee\left(\bigvee_{i=1}^{n-1} J_{i}(a)\right)=1$.

2. From Axiom 2.3, it follows that $J_{i}(a) \wedge J_{j}(a)=\bigwedge_{k \neq i} \neg J_{k}(a) \wedge J_{j}(a)=0$. 
3. Suppose $J_{i}(a)=1$ and let $j \neq i . \quad J_{j}(a)=\bigwedge_{k \neq j} \neg J_{k}(a) \leq \neg J_{i}(a)=$ $\neg 1=0$.

4. It follows from item 3 and Axiom 2.9.

5. $J_{0}(0)=J_{0}(\neg 1)=J_{0}\left(\neg J_{n-1}(1)\right)$

$$
=\bigvee_{\neg \frac{i}{n-1}=0} J_{i}\left(J_{n-1}(1)\right) \geq J_{n-1}\left(J_{n-1}(1)\right)=1 \text {. }
$$

From this and Axiom 2.9 we obtain 5.

Lemma 2.14 Let $\mathbf{A}$ be an $L_{n}$-algebra for the matrix $\mathbf{M}$. The following properties hold for any $a, b$ in $A$ :

1. $J_{n-1}(a \vee b)=J_{n-1}(a) \vee J_{n-1}(b)$,

2. $J_{n-1}(a \wedge b)=J_{n-1}(a) \wedge J_{n-1}(b)$.

\section{Proof}

1. From Axioms $2.8\left(\mathrm{Cn}_{\vee}\right)$ and $\left(\mathrm{C}_{\vee}\right)$ of Definition 2.1 and $\bigvee_{i=0}^{n-1} J_{i}(c)=1$ we obtain that

$$
\begin{aligned}
J_{n-1}(a \vee b) & =\bigvee_{\frac{i}{n-1} \vee \frac{j}{n-1}=1}\left(J_{i}(a) \wedge J_{j}(b)\right) \\
& =\left(\bigvee_{j=0}^{n-1}\left(J_{n-1}(a) \wedge J_{j}(b)\right)\right) \vee\left(\bigvee_{i=0}^{n-1}\left(J_{i}(a) \wedge J_{n-1}(b)\right)\right) \\
& =J_{n-1}(a) \vee J_{n-1}(b) .
\end{aligned}
$$

2. From Axiom $\left(\mathrm{C}_{\wedge}\right)$ it follows that

$$
J_{n-1}(a \wedge b)=\bigvee_{\frac{i}{n-1} \wedge \frac{j}{n-1}=1}\left(J_{i}(a) \wedge J_{j}(b)\right)=J_{n-1}(a) \wedge J_{n-1}(b) .
$$

Lemma 2.15 Let $\mathbf{A}$ be an $L_{n}$-algebra for $\mathbf{M}$. For any $a, b, c$ in $A$ one has

$\mathrm{C}_{\wedge} \quad a \wedge b=1$ iff $a=1$ and $b=1$,

$\mathrm{C}_{\supset 1} \quad a \supset(b \supset a)=1$,

$\mathrm{C}_{\supset 2} \quad(a \supset(b \supset c)) \supset((a \supset b) \supset(a \supset c))=1$,

$\mathrm{C}_{\supset 3} \quad 1 \supset a=1$ iff $a=1$.

Proof

$\left(\mathrm{C}_{\wedge}\right) \quad$ Let $a, b$ be such that $a \wedge b=1$. It is enough to see that $J_{n-1}(a)=J_{n-1}(b)=1$.

$$
1=J_{n-1}(a \wedge b)=\bigvee_{\frac{i_{1}}{n-1} \wedge \frac{i_{2}}{n-1}=1} J_{i_{1}}(a) \wedge J_{i_{2}}(b)=J_{n-1}(a) \wedge J_{n-1}(b) .
$$

Since $E(\mathbf{M})$ is a Boolean algebra, it follows that $J_{n-1}(a)=J_{n-1}(b)=1$. The other implication is easier.

$\left(\mathrm{C}_{\supset 1}\right)$ From Axiom 2.8,

$$
\begin{aligned}
& J_{n-1}(a \supset(b \supset a))=\bigvee_{\frac{i_{1}}{n-1} \supset \frac{i_{2}}{n-1}=1} J_{i_{1}}(a) \wedge J_{i_{2}}(b \supset a)
\end{aligned}
$$

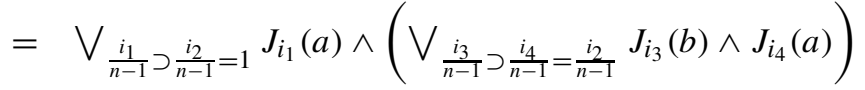

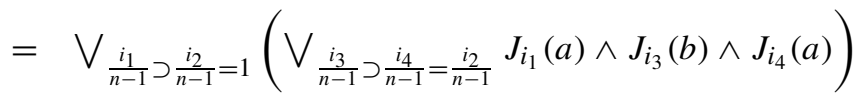

$$
\begin{aligned}
& =\alpha_{1} \text {. }
\end{aligned}
$$


From Lemma 2.13, $J_{i_{1}}(a) \wedge J_{i_{4}}(a)=0$ for $i_{1} \neq i_{4}$, and then

$$
\begin{aligned}
\alpha_{1} & =\bigvee_{\frac{i_{1}}{n-1} \supset \frac{i_{2}}{n-1}=1}\left(\bigvee_{\frac{i_{3}}{n-1} \supset \frac{i_{4}}{n-1}=\frac{i_{2}}{n-1}} J_{i_{1}}(a) \wedge J_{i_{3}}(b)\right) \\
& =\bigvee_{\frac{i_{3}}{n-1} \supset \frac{i_{4}}{n-1}=\frac{i_{2}}{n-1}}\left(\bigvee_{\frac{i_{1}}{n-1} \supset \frac{i_{2}}{n-1}=1} J_{i_{1}}(a) \wedge J_{i_{3}}(b)\right) \\
& =\alpha_{2} .
\end{aligned}
$$

$\operatorname{Recall}\left(\mathrm{C}_{\supset 1}\right): \frac{i_{1}}{n-1} \supset\left(\frac{i_{3}}{n-1} \supset \frac{i_{1}}{n-1}\right)=1$, then

$$
\begin{aligned}
\alpha_{2} & =\bigvee_{\frac{i_{1}}{n-1} \supset\left(\frac{i_{3}}{n-1} \supset \frac{i_{1}}{n-1}\right)=1} J_{i_{1}}(a) \wedge J_{i_{3}}(b) \\
& =\bigvee_{i_{1} j} J_{i}(a) \wedge J_{j}(b)=\bigvee_{i} J_{i}(a) \wedge \bigvee_{j} J_{j}(b) \\
& =1
\end{aligned}
$$

$\left(\mathrm{C}_{\supset 2}\right)$ The proof is similar to the previous one.

$\left(\mathrm{C}_{\supset 3}\right)$ In order to prove that $1 \supset a=1$ implies $a=1$ observe that it is enough to see $J_{n-1}(1 \supset a)=1$ implies $J_{n-1}(a)=1$. From Axiom 2.8 it follows that

$$
\begin{aligned}
J_{n-1}(1 \supset a) & =\bigvee_{\frac{i_{1}}{n-1} \supset \frac{i_{2}}{n-1}=1} J_{i_{1}}(1) \wedge J_{i_{2}}(a) \\
& =\bigvee_{1 \supset \frac{i_{2}}{n-1}=1} J_{1}(1) \wedge J_{i_{2}}(a) \\
& =\bigvee_{1 \supset \frac{i_{2}}{n-1}=1} J_{i_{2}}(a) .
\end{aligned}
$$

From the analogous property for $\mathbf{M}, J_{n-1}(1 \supset a)=J_{n-1}(a)$. Since from hypothesis $J_{n-1}(1 \supset a)=1$, then $J_{n-1}(a)=1$.

Notice that the condition $\left(\mathrm{C}_{\vee}\right)$ does not hold in general. Indeed, it is not true that in a Boolean algebra $x \vee y=1$ implies $x=1$ or $y=1$.

\subsection{Examples}

Example 2.16 Let $M_{3}=\left\{0, \frac{1}{2}, 1\right\}$ and let the operations on $M_{3}$ be given by

$$
\begin{gathered}
x \wedge y=\left\{\begin{array}{ll}
1 & \text { if } x=1 \text { and } y=1 \\
0 & \text { otherwise, }
\end{array} \quad x \vee y= \begin{cases}1 & \text { if } x=1 \text { or } y=1 \\
0 & \text { otherwise, }\end{cases} \right. \\
\neg x=\left\{\begin{array}{ll}
1 & \text { if } x=0 \text { or } x=\frac{1}{2} \\
0 & \text { if } x=1,
\end{array} \quad x \supset y=\neg x \vee y .\right.
\end{gathered}
$$

It is easy to check that $\mathbf{M}=\left\langle M_{3}, \mathbb{F}\right\rangle$ is an $L_{n}$-algebra for $\mathbf{M}$ for this 3-matrix $\mathbf{M}$.

In the following examples, we consider the natural order on $M_{n}$, that is, $0<\frac{1}{n-1}<\cdots<\frac{n-2}{n-1}<1$ and its natural additive structure.

Example 2.17 Consider now $M_{3}$ with the operations

$$
\begin{aligned}
& x \wedge y=\min \{x, y\}, \quad x \vee y=\max \{x, y\}, \\
& \neg x=1-x, \quad x \supset y=\min \{1,1-x+y\} .
\end{aligned}
$$

Then $\mathbf{M}=\left\langle M_{3}, \mathbb{F}\right\rangle$ is an $L_{n}$-algebra for $\mathbf{M}$ for this 3-matrix $\mathbf{M}$. 
Example 2.18 Recall (see Cignoli and de Gallego [6], Boicescu et al. [4]) that an $n$-valued Moisil algebra is a system $\left\langle A, \vee, \wedge, \neg, \sigma_{1}, \ldots, \sigma_{n}, 0,1\right\rangle$ such that $\langle A, \vee, \wedge, 0,1\rangle$ is a distributive lattice with unit 1 and zero 0 , and $\neg, \sigma_{1}, \ldots, \sigma_{n}$ are unary operators defined on $A$, fulfilling certain conditions. In any $n$-valued Moisil algebra, there are operators $J_{0}, \ldots, J_{n-1}$ such that they can be written in terms of the operators $\sigma_{1}, \ldots, \sigma_{n}$ :

$$
J_{i}(x)=\sigma_{n-1}(x) \wedge \neg \sigma_{n-i-1}(x) .
$$

Moreover, the operators $\sigma_{1}, \ldots, \sigma_{n}$ can be expressed in terms of $J_{0}, \ldots, J_{n-1}$ :

$$
\sigma_{i}(x)=\bigvee_{j=1}^{i} J_{n-j}(x) .
$$

Now it is convenient to take an $n$-valued Moisil algebra to be a system

$$
\left\langle A, J_{0}, \ldots, J_{n-1}, \neg, \vee, \wedge, \supset, \sigma_{1}, \ldots, \sigma_{n-1}\right\rangle ;
$$

the operation $\supset$ is now the weak implication:

$$
x \supset y=\sigma_{n-1}(\neg x) \vee y .
$$

Let $M_{n}=\left\{\frac{j}{n-1}: j=0, \ldots, n-1\right\}$, the $n$-valued Moisil algebra with the natural lattice operations $\vee, \wedge, 0,1, \neg$, and $\sigma_{i}$ given by

$$
\neg \frac{j}{n-1}=1-\frac{j}{n-1}, \quad \sigma_{i}\left(\frac{j}{n-1}\right)=\left\{\begin{array}{cc}
0 & \text { if } i+j<n \\
1 & \text { if } i+j \geq n .
\end{array}\right.
$$

Let $\mathbf{M}_{n}=\left\langle M_{n}, \mathbb{F}\right\rangle$ be an algebra of type $\mathbb{F}$. Since conditions $\left(\mathrm{C}_{J_{i}}\right),\left(\mathrm{C}_{\wedge}\right), \ldots,\left(\mathrm{C}_{\supset 3}\right)$ are satisfied, from Lemma 2.11 it follows that $\mathbf{M}_{n}$ is an $L_{n}$-algebra for the matrix $\mathbf{M}_{n}$.

Remark 2.19 Let $\mathbf{M}_{n}$ be as above. Observe that if $n=4$ and $\supset$ is the Łukasiewicz implication,

$$
x \supset y=\min \{1,1-x+y\}
$$

and

$$
\neg x=1-x, \quad x \vee y=\max \{x, y\}, \quad x \wedge y=\min \{x, y\} .
$$

In this case, $\mathbf{M}_{4}$ is not an $L_{n}$-algebra for the matrix $\mathbf{M}_{4}$. It is enough to observe that the Eukasiewicz implication does not verify $\left(C_{\supset 2}\right)$ :

$$
\left(\frac{1}{3} \supset\left(\frac{2}{3} \supset 0\right)\right) \supset\left(\left(\frac{1}{3} \supset \frac{2}{3}\right) \supset\left(\frac{1}{3} \supset 0\right)\right) \neq 1 .
$$

Example 2.20 Let $\mathbf{M}_{6}=\left\langle M_{6}, \mathbb{F}\right\rangle$ be the matrix given in Example 2.18 for $n=6$. Let $A=\left\{0, \frac{1}{3}, \frac{2}{3}, 1\right\}$ and let $\mathbb{F}=\left\{\bar{J}_{0}, \ldots, \bar{J}_{5}, \neg, \vee, \wedge, \supset\right\}$ such that $\neg, \vee, \wedge$ are the same as in the same example, $\supset$ is the weak implication, and $\overline{J_{0}}, \ldots, \overline{J_{5}}$ are given by

$$
\left[\begin{array}{ccccccc} 
& \overline{J_{0}} & \overline{J_{1}} & \overline{J_{2}} & \overline{J_{3}} & \overline{J_{4}} & \overline{J_{5}} \\
0 & 1 & 0 & 0 & 0 & 0 & 0 \\
\frac{1}{3} & 0 & 1 & 0 & 0 & 0 & 0 \\
\frac{2}{3} & 0 & 0 & 0 & 0 & 1 & 0 \\
1 & 0 & 0 & 0 & 0 & 0 & 1
\end{array}\right]
$$


Let $\mathbf{A}=\langle A, \mathbb{F}\rangle$. Then $\mathbf{A}$ is an $L_{6}$-algebra for the matrix $\mathbf{M}_{6}$. (See [6]). We are going to check $\overline{J_{0}}(\neg a)=\bigvee_{\neg \frac{i}{n-1}=0} \overline{J_{i}}(a)$. Observe that

$$
\overline{J_{0}}(\neg a)= \begin{cases}0 & \text { if } a \in\left\{0, \frac{1}{3}, \frac{2}{3}\right\} \\ 1 & \text { if } a=1\end{cases}
$$

and also

$$
\bigvee_{\neg \frac{i}{5}=0} \overline{J_{i}}(a)=\overline{J_{5}}(a)=\overline{J_{0}}(\neg a) .
$$

Analogously for $\overline{J_{1}}(\neg a), \ldots, \overline{J_{5}}(\neg a)$.

In order to check this axiom for the operation $\supset$, recall that the weak implication on $\left\{0, \frac{1}{3}, \frac{2}{3}, 1\right\}$ is given by

$$
x \supset y=\sigma_{3}(\neg x) \vee y=\left\{\begin{array}{cc}
1 & \text { if } x \neq 1 \\
y & \text { if } x=1,
\end{array}\right.
$$

and the weak implication on $\left\{0, \frac{1}{5}, \frac{2}{5}, \frac{3}{5}, \frac{4}{5}, 1\right\}$ is given by

$$
x \supset y=\sigma_{5}(\neg x) \vee y .
$$

For example, we are going to check that $\overline{J_{0}}\left(a_{1} \supset a_{2}\right)=\bigvee_{\frac{i_{1}}{5} \supset \frac{i_{2}}{5}=0} \overline{J_{i_{1}}}\left(a_{1}\right) \wedge \overline{J_{i_{2}}}\left(a_{2}\right)$ : $\overline{J_{0}}\left(a_{1} \supset a_{2}\right)=1$ if and only if $a_{1} \supset a_{2}=0$ if and only if $a_{1}=1$ and $a_{2}=0$. On the other hand,

$$
\bigvee_{\frac{i_{1}}{5} \supset \frac{i_{2}}{5}=0} \overline{J_{i_{1}}}\left(a_{1}\right) \wedge \overline{J_{i_{2}}}\left(a_{2}\right)=\overline{J_{5}}\left(a_{1}\right) \wedge \overline{J_{0}}\left(a_{2}\right)=1
$$

if and only if $a_{1}=1$ and $a_{2}=0$. In the same way we can prove the other cases.

Remark 2.21 Recall that the intuitionist implication is defined by

$$
x \supset y=\min \{z: x \wedge z \leq y\} .
$$

On $\left\{0, \frac{1}{n-1}, \ldots, 1\right\}, \supset$ is given by

$$
x \supset y= \begin{cases}1 & \text { if } x \leq y \\ y & \text { otherwise. }\end{cases}
$$

It is known-see [6] - that this implication can be expressed in terms of the operations $\sigma_{i}$. This implication can be used in place of the weak one in the previous example, obtaining another example of $n$-matrix.

Example 2.22 Let $\mathbb{F}=\left\{J_{0}, J_{1}, \neg, \vee, \wedge, \supset,\right\}, \mathbf{M}_{2}=\langle\{0,1\}, \mathbb{F}\rangle$ such that $\langle\{0,1\}, \neg, \vee, \wedge, \supset\rangle$ is a Boolean algebra. Let $\mathbf{A}$ be an $L_{n}$-algebra for the matrix $\mathbf{M}_{2}$. Then $\mathbf{A}$ is also a Boolean algebra. For example, to check the axiom $a \vee \neg a$ it is enough to prove that $J_{1}(a \vee \neg a)=1$. First observe that $J_{1}(\neg a)=\vee_{\neg i=1} J_{i}(a)=J_{0}(a)$ and from Lemma 2.14,

$$
J_{1}(a \vee \neg a)=J_{1}(a) \vee J_{1}(\neg a)=J_{1}(a) \vee J_{0}(a)=1 .
$$

In the same way it is easy to prove all the axioms.

Example 2.23 Let $\mathbf{A} \in L_{n} M$ be the variety of the monadic $n$-valued Łukasiewicz algebras—see Abad [1]. First recall that the only operator $\exists$ on $\left\{0, \frac{1}{n-1}, \ldots, \frac{n-2}{n-1}, 1\right\}$ is the identity. Then, if $\mathbf{A}$ is an $L_{n}$-algebra for a matrix $\mathbf{M}$ as in Example 2.18 and $G=\{\exists\}$, from Remark 2.12 it must be that $\exists=\operatorname{Id}_{A}$. It follows that in general if $\mathbf{A}$ $\in L_{n} M$, then $\mathbf{A}$ is not an $L_{n}$-algebra. 


\section{Boolean Congruences}

As usual, a congruence $\theta$ on an algebra $\mathbf{A}=\langle A, \mathbb{F}\rangle$ is an equivalence relation that is compatible with the operations belonging in $\mathbb{F}$. In particular, $a \theta b$ implies $J_{i}(a) \theta J_{i}(b), i=0, \ldots, n-1$. Let $\theta_{e}$ be the restriction of $\theta$ to the Boolean algebra $E(\mathbf{A})$. Notice that if $\eta$ is a congruence on the Boolean algebra $E(\mathbf{A})$, then from Axioms $\left(\mathrm{Cn}-\mathrm{J}_{0}\right),\left(\mathrm{Cn}-\mathrm{J}_{n-1}\right)$, and $\left(\mathrm{Cn}-\mathrm{J}_{i}\right)$, we have that $\eta$ also preserves the connectives $J_{i}: J_{j}(a) \eta J_{k}(b)$ implies that $J_{i}\left(J_{j}(a)\right) \eta J_{i}\left(J_{k}(b)\right)$.

Lemma 3.1 For each congruence $\eta$ on $E(\mathbf{A})$, let $\bar{\eta}$ be the relation on $A$ such that $a \bar{\eta} b$ if and only if $J_{i}(a) \eta J_{i}(b), i=0, \ldots, n-1$. Then $\bar{\eta}$ is a congruence on $A$.

Proof It is obvious that $\bar{\eta}$ is an equivalence relation on $A$ and it follows from Axiom 2.8 that it also preserves the operations in $F$.

Notice that for each congruence $\theta$ on $A$ we have that $\theta \subseteq \overline{\theta_{e}}$ and $\eta \subseteq(\bar{\eta})_{e}$.

Definition 3.2 Let $\theta$ be a congruence on $A$. We say that $\theta$ is a Boolean congruence if and only if $\theta=\overline{\theta_{e}}$.

Let $\mathbf{A} \in \mathcal{M}$. We denote by $\operatorname{Con}(A)$ the complete lattice of congruences of $\mathbf{A}$. Following Pigozzi [7], we say that $\theta \in \operatorname{Con}(A)$ is an $\mathcal{M}$-congruence provided that $A / \theta \in \mathcal{M}$. The set of $\mathcal{M}$-congruences of $\mathbf{A}$ is a complete lattice of $\operatorname{Con}(A)$ that we denote $\operatorname{Con}_{\mathcal{M}}(A)$.

Lemma 3.3 The Boolean congruences are exactly the $\mathcal{M}$-congruences.

Proof Suppose that $A / \theta \in \mathcal{M}$. We need to show that $\overline{\theta_{e}} \subseteq \theta$ : let $a, b \in A$ such that $a \overline{\theta_{e}} b$. Then $J_{i}(a) \theta J_{i}(b)$ for each $i=0, \ldots, n-1$. Since Axiom 2.9 holds in $A / \theta$ we have that $a \theta b$. For the converse, suppose that $J_{i}(a) \theta J_{i}(b)$ for $i=0, \ldots, n-1$. Then we have that $J_{i}(a) \theta_{e} J_{i}(b)$ for $i=0, \ldots, n-1$ and then $a \overline{\theta_{e}} b$. Since $\theta=\overline{\theta_{e}}$ it follows that $a \theta b$.

Lemma 3.4 If $\theta$ is a maximal congruence on $\mathbf{A}$, then $\theta_{e}$ is a maximal congruence on $E(\mathbf{A})$, or it is the universal congruence on $E(\mathbf{A})$.

Proof If $\theta_{e} \subseteq \eta$, then $\theta \subseteq \overline{\theta_{e}} \subseteq \bar{\eta}$. Since $\theta$ is maximal, then $\bar{\eta}=\theta$ or $\bar{\eta}=A \times A$. In the first case, $\eta \subseteq(\bar{\eta})_{e}=\theta_{e} \subseteq \eta$, therefore $\theta_{e}=\eta$. In the case $\bar{\eta}=A \times A$, then it is immediate that $\eta=E(\mathbf{A}) \times E(\mathbf{A})$.

Lemma 3.5 Let $U$ be an ultrafilter in $E(\mathbf{A})$. Then, for each $a \in A$, there is $a$ unique $i \in\{0, \ldots, n-1\}$ such that $J_{i}(a) \in U$.

Proof It follows immediately from Lemma 2.13.

It is well known that, in a Boolean algebra, filters can be identified with congruences. We denoted $U_{\eta}$, the filter associated with the congruence $\eta$, and $\eta_{U}$, the congruence associated with the filter $U$. Also we put $[x]=\{y: y \eta x\}$.

Lemma 3.6 Let $U$ be as above. If $J_{i}(a) \in U$ then $J_{n-1}\left(J_{i}(a)\right) \in U$, else $J_{0}\left(J_{i}(a)\right) \in U$.

Proof In the first case, observe that $J_{i}(a)=J_{n-1}\left(J_{i}(a)\right) \in U$. If $J_{i}(a) \notin U$ then $\neg J_{i}(a)=J_{0}\left(J_{i}(a)\right) \in U$. 
Theorem 3.7 Let $\mathbf{A}$ be an $L_{n}$-algebra for the matrix $\mathbf{M}$. Let $\eta$ be a maximal congruence on $E(\mathbf{A})$. There is a homomorphism $f_{\eta}: A / \bar{\eta} \hookrightarrow M_{n}$, that is, the quotient $A / \bar{\eta}$ is isomorphic to a subalgebra of $M_{n}$.

Proof Note first that since $\bar{\eta}$ is a Boolean congruence, $A / \bar{\eta} \in \mathcal{M}$. Let $U_{\eta}$ be the prime filter on $E(\mathbf{A})$ as above, and let

$$
f_{\eta}: A / \bar{\eta} \rightarrow M_{n}
$$

given by

$$
f_{\eta}([a])=\frac{i}{n-1}
$$

where $i$ is the unique integer such that $J_{i}(a) \in U_{\eta}$. The function $f_{\eta}$ is well defined since if $[a]=[b]$, then $J_{i}(a) \eta J_{i}(b)$ for each $i=0, \ldots, n-1$, and hence $J_{i}(a) \in U_{\eta}$ if and only if $J_{i}(b) \in U_{\eta}$.

Let us check that $f_{\eta}$ is a homomorphism: let

$$
* \in \mathbb{F}-\left\{J_{i}, i=0, \ldots, n-1\right\}, a_{1}, a_{2}, \ldots, a_{k}
$$

belong in $A$, and suppose that

$$
f_{\eta}\left(\left[a_{j}\right]\right)=\frac{i_{j}}{n-1}, j=0, \ldots, k
$$

that

and that

$$
\frac{r}{n-1}=*\left(\frac{i_{1}}{n-1}, \frac{i_{2}}{n-1}, \ldots, \frac{i_{k}}{n-1}\right) \text {, }
$$

$$
J_{i}\left(*\left(a_{1}, a_{2}, \ldots, a_{k}\right)\right) \in U_{\eta} .
$$

Then $f_{\eta}\left(*\left(\left[a_{1}\right],\left[a_{2}\right], \ldots,\left[a_{k}\right]\right)\right)=f_{\eta}\left(\left[*\left(a_{1}, a_{2}, \ldots, a_{k}\right)\right]\right)=\frac{i}{n-1}$, and in order to prove $f_{\eta}\left(*\left(\left[a_{1}\right],\left[a_{2}\right], \ldots,\left[a_{k}\right]\right)\right)=*\left(f_{\eta}\left(\left[a_{1}\right]\right), f_{\eta}\left(\left[a_{2}\right]\right), \ldots, f_{\eta}\left(\left[a_{k}\right]\right)\right)$ we must show that $J_{r}\left(*\left(a_{1}, a_{2}, \ldots, a_{k}\right)\right) \in U_{\eta}$. We have

$$
\begin{aligned}
J_{r}\left(*\left(a_{1}, a_{2}, \ldots, a_{k}\right)\right) & =\bigvee_{*\left(\frac{j_{1}}{n-1}, \frac{j_{2}}{n-1}, \ldots, \frac{j_{k}}{n-1}\right)=\frac{r}{n-1}} \bigwedge_{l=1}^{k} J_{j_{l}}\left(a_{l}\right) \\
& \geq\left(J_{i_{1}}\left(a_{1}\right) \wedge \cdots \wedge J_{i_{k}}\left(a_{k}\right)\right) \in U_{\eta},
\end{aligned}
$$

because each $J_{i_{l}}\left(a_{l}\right) \in U_{\eta}$. Also $f_{\eta}\left(J_{j}([a])\right)=J_{j}\left(f_{\eta}([a])\right)$ for each $j=0, \ldots, n-1$. Indeed, let $i$ be such that $J_{i}(a) \in U_{\eta}$. Hence $J_{j}\left(f_{\eta}([a])\right)=J_{j}\left(\frac{i}{n-1}\right)=1$ if and only if $i=j$, and the result follows from Lemma 3.6. Finally, $f_{\eta}$ is injective: if $(a, b) \notin \bar{\eta}$ then $\left(J_{j}(a), J_{j}(b)\right) \notin \eta$ for some $j$. Either $J_{j}(a) \in U_{\eta}$ and $J_{j}(b) \notin U_{\eta}$, or $J_{j}(a) \notin U_{\eta}$ and $J_{j}(b) \in U_{\eta}$. In both cases $f_{\eta}([a]) \neq f_{\eta}([b])$.

Lemma 3.8 Let I be the set of maximal elements of $\operatorname{Con}_{\mathcal{M}}(\mathbf{A})$. Then $\cap I=\Delta$ is the minimum element of $\operatorname{Con}_{\mathcal{M}}(\mathbf{A})$.

Proof Suppose $a \neq b$, then there is an $i$ such that $J_{i}(a) \neq J_{i}(b)$. Let $U$ be an ultrafilter such that $J_{i}(a) \in U$ and $J_{i}(b) \notin U$. Then $\left(J_{i}(a), J_{i}(b)\right) \notin \eta_{U}$, and $(a, b) \notin \overline{\eta_{U}}$. It follows that $(a, b) \notin \bigcap\{\bar{\eta} \in I\}$.

From the above lemma, Theorem 3.7, and a well-known theorem of Birkhoff (see, for instance, Burris and Sankappanavar [5, Chapter 2, Theorem 8.6]) we obtain another proof of Theorem 5.20 given in [2].

Theorem 3.9 Let $\mathbf{A}$ be an $L_{n}$-algebra for the matrix $\mathbf{M}$. Then $\mathbf{A}$ is a subdirect product of a family of subalgebras of the matrix $\mathbf{M}$. 


\subsection{Examples}

Example 3.10 Let $\mathbf{M}_{n}$ be an $n$-matrix; since $E\left(\mathbf{M}_{n}\right)=\{0,1\}$, the set $\operatorname{Con}_{\mathcal{M}\left(M_{n}\right)}\left(\mathbf{M}_{n}\right)$ has exactly two elements. (Observe that, if $\mathbf{A} \in \mathcal{M}$ and $\Delta_{\mathbf{A}}=\min \operatorname{Con}(\mathbf{A})$, from Axiom 2.9 it follows that $\overline{\Delta_{E\left(M_{n}\right)}}=\Delta_{M_{n}}$.) Then $M_{n} / \bar{\eta}=M_{n}$ or $M_{n} / \bar{\eta}$ has only one element. However, $\mathbf{M}_{n}$ may be not simple. Indeed, let $\mathbf{M}_{n}$ be as in Example 2.16. Then $\theta=\Delta_{M_{n}} \cup\{(0,1),(1,0)\}$ verifies $\Delta_{M_{n}} \varsubsetneqq \theta \varsubsetneqq M_{n} \times M_{n}$, and $\theta \notin \operatorname{Con}_{\mathcal{M}\left(M_{n}\right)}\left(\mathbf{M}_{n}\right)$.

Example 3.11 Let $F=\left\{J_{0}, J_{1}, \ldots, J_{n-1}\right\} \cup\{\neg, \vee, \wedge, \supset\}, G=\left\{\sigma_{1}, \ldots, \sigma_{n}\right\}$, $\mathbb{F}=F \cup G, \mathbb{F}^{\prime}=\{\neg, \vee, \wedge\} \cup G$. Let $\mathbf{M}_{n}=\left\langle M_{n}, \mathbb{F}\right\rangle$ be the matrix such that $\mathbf{M}_{n}^{\prime}=\left\langle M_{n}, \mathbb{F}^{\prime}\right\rangle$ is an $n$-Moisil algebra (see Example 2.18). Since in this case the quasi identity given by Axiom 2.9 can be replaced by identities (see [4]) then $\mathcal{M}$ is a variety.

\section{An Axiomatization of Anshakov-Rychkov $n$-valued Propositional Calculi}

Let $\mathbf{M}$ be an $n$-matrix of type $\mathbb{F}$ as in Section 2. Now we are going to define the $n$ valued propositional calculus of Anshakov-Rychkov for the matrix $\mathbf{M}$. Let $\mathcal{F}$ denote the algebra of well-formed formulas constructed in the usual way from a denumerable set $\left\{p_{m}\right\}_{m \in N}$ of propositional variables by means of the connectives in $\mathbb{F}$.

Let $E(\mathcal{F}) \subseteq \mathcal{F}$ be the set of external formulas which are defined inductively as follows:

1. for each $i=1, \ldots, n-1$, if $X$ is a formula then $J_{i} X$ is an external formula;

2. if $X$ and $Y$ are external formulas, $\neg X, X \vee Y, X \wedge Y, X \supset Y$ are external formulas.

Notation 4.1 $X \subsetneq Y=(X \supset Y) \wedge(Y \supset X), T=J_{n-1}\left(p_{n}\right) \vee \neg J_{n-1}\left(p_{n}\right)$.

The Axiom schemes are the following:

(Boolean Axioms) If $\Phi$ is a classical tautology, let $B_{\Phi}$ be the formula in which we substitute each appearance of each propositional variable by any formula of the form $J_{i}(X)$. Then $B_{\Phi}$ is an axiom of this calculi. For example, $J_{i}(X) \vee \neg J_{i}(X)$ is an axiom, and for any $X, Y, J_{i}(X) \supset\left(J_{i}(Y) \supset J_{i}(X)\right)$ and

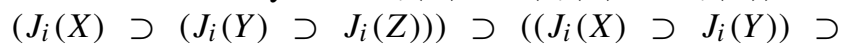
$\left.\left(J_{i}(X) \supset J_{i}(Z)\right)\right)$ are axioms.

$n-\mathrm{Val}_{i}$

$J_{i}(X) \stackrel{\ulcorner}{\supset} \bigwedge_{j \neq i} \neg J_{j}(X), i=0, \ldots, n-1$

$\mathrm{CJ}_{i}$$$
\begin{cases}\mathrm{CJ}_{0} & J_{0}\left(J_{i}(X)\right) \stackrel{\subset}{\supset} \neg J_{i}(X) \\ \mathrm{CJ}_{n-1} & J_{n-1}\left(J_{i}(X)\right) \stackrel{\ulcorner}{\supset} J_{i}(X) \\ \mathrm{CJ}_{j} & J_{j}\left(J_{i}(X)\right) \stackrel{\subset}{\supset} \neg, j \notin\{0, n-1\}\end{cases}
$$

$\mathrm{Cl}$

$$
\left\{\begin{array}{rr}
\mathrm{Cl}^{-J_{*}} & J_{i}(X) * J_{j}(Y) \stackrel{\subsetneq}{\supset} J_{n-1}\left(J_{i}(X) * J_{j}(Y)\right), \\
& * \in\{\vee, \wedge, \supset\} \\
\mathrm{Cl}^{-J} & \neg J_{n-1}\left(J_{i}(X)\right) \stackrel{\ulcorner}{\supset} J_{n-1}\left(\neg J_{i}(X)\right)
\end{array}\right.
$$

Cn-G

$$
\begin{array}{r}
J_{i}\left(G\left(X_{1}, \ldots, X_{m}\right)\right) \stackrel{\ulcorner}{\supset} \bigvee_{G\left(\frac{i_{1}}{n-1}, \ldots, \frac{i_{m}}{n-1}\right)=\frac{i}{n-1}} \bigwedge_{j=1}^{m} J_{i_{j}}\left(X_{j}\right), \\
G \in \mathbb{F} \backslash\left\{J_{i}, i=0, \ldots, n-1\right\}
\end{array}
$$


(Rules of inference) $\begin{cases}\text { modus ponens: } & \frac{J_{i}(X),}{n_{i}(X) \supset J_{j}(Y)} \\ \text { J-introduction: } & \frac{X}{J_{j}(Y)} \\ \text { J-elimination: } & \frac{J_{n-1}(X)}{X}\end{cases}$

We denote by $\&(\mathbf{M})$, or simply by $\&$, the logic given by this language and these axioms and inference rules. $\&$ is the Anshakov-Rychkov propositional logic for $\mathbf{M}$.

If $D$ is a unary predicate, $s_{D}$ is the extension of $\delta$ to which we add the symbol $D$. Let $\Gamma \subseteq \mathcal{F}, X \in \mathcal{F}$. Derivability, theorem, and theory are defined in the usual way, and the notation ' $\Gamma \vdash X$ ' means that ' $X$ is derivable from $\Gamma$ '. Let $\mathcal{M}(\mathbf{M})$ be the quasi variety of algebras defined in Remark 2.10. Let $\mathbf{A} \in \mathcal{M}$.

A valuation on $\mathbf{A}$ is a function $v: \mathcal{F} \rightarrow \mathbf{A}$ that preserves the connectives belonging to $\mathbb{F} . v(X)$ is an interpretation of the formula $X$.

Notation 4.2 As usual, $\Gamma \vDash_{\mathbf{A}, v} X$ if and only if $v(\Gamma) \subseteq\{1\}$ implies $v(X)=1$, and $\Gamma \vDash_{\mathbf{A}} X$ if and only if for each valuation $v$ on $\mathbf{A}, \Gamma \vDash_{\mathbf{A}, v} X$. If $\Gamma=\varnothing, X$ is a tautology for $\mathbf{A}$ and we write $\vDash_{\mathbf{A}} X$. Also, we write $\Gamma \vDash_{\mathcal{M}} X$ (or simply $\Gamma \vDash X$ ) if and only if $\Gamma \vDash_{\mathbf{A}} X$ for each $\mathbf{A} \in \mathcal{M}$.

$X$ is a tautology for $\mathcal{M}$ if and only if $X$ is a tautology for $\mathbf{A}$, for each $\mathbf{A} \in \mathcal{M}$. In this case, we write $\vDash X$. Finally, if $X$ is a formula with a single variable $p$, then $X[Y / p]$ is the formula obtained from $X$ by replacing each appearance of $p$ by $Y$.

Let $\&$ be the Anshakov-Rychkov propositional logic for $\mathbf{M}$, and $\mathcal{M}=\mathcal{M}(\mathbf{M})$.

Theorem 4.3 \& is algebraizable with equivalent algebraic semantic $\mathcal{M}$. This means (see [3], Definition 2.8) that

1. there exists a finite system of equations-called defining equations for $\&$ and $\mathcal{M}, \delta_{i}(p) \approx \epsilon_{i}(p)$ for $i<m$-with a single variable $p$ such that for all $\Gamma \cup\{X\} \subseteq \mathcal{F}$ and each $j<s, \Gamma \vdash X$ if and only if $\left\{\delta_{i}(Y / p) \approx \epsilon_{i}(Y / p): j<s, Y \in \Gamma\right\} \vDash \delta_{j}(X / p) \approx \epsilon_{j}(X / p) ;$

2. there exists a finite system $\Delta_{k}(p, q)$, for $k<t$ offormulas with two variables such that, for every equation $X \approx Y, X \approx Y \vDash \delta(X \Delta Y) \approx \epsilon(X \Delta Y)$ and $\delta(X \Delta Y) \approx \epsilon(X \Delta Y) \vDash X \approx Y$.

In order to prove Theorem 4.3, we will use the following result by Blok and Pigozzi.

Theorem 4.4 ([3], Theorem 5.1) The following statements are equivalent.

1. $\&$ is algebraizable with equivalent semantics $\mathcal{M}$.

2. For every algebra $\mathbf{A}$ the Leibnitz operator $\Omega_{\mathbf{A}}$ is an isomorphism between the lattices of \&-filters and $\mathcal{M}$-congruences of $\mathbf{A}$.

We know that the $\mathcal{M}$-congruences of $A$ are exactly the Boolean congruences. In order to prove Theorem 4.4 we first study the class of $\&$-filters. Recall that a subset $F$ of $\mathbf{A}$ is an $\&$-filter when $F$ contains all the interpretations of the logical axioms of $\delta$ and is closed under each inference rule: this means that if the premises are in $F$, then the conclusion also belongs to $F$.

Let $U$ be a filter on the Boolean algebra $E(\mathbf{A}) . F$ is a Boolean filter if and only if $F=\left\{a \in A: J_{n-1}(a) \in U\right\}$.

Lemma 4.5 The following statements are equivalent. 
1. F is a Boolean filter.

2. $F$ is an \&-filter.

Proof Let $F=\left\{a \in A: J_{n-1}(a) \in U\right\}$. Since $1 \in F, F$ contains the interpretations of the logical axioms. Now let $J_{i}(a) \in F$ and $J_{i}(a) \supset J_{j}(b) \in F$. Recalling Axiom $2.4\left(\mathrm{Cn}_{-} \mathrm{J}_{n-1}\right)$, note that for any $J_{i}(a) \in E(\mathbf{A}), J_{i}(a) \in F$ if and only if $J_{i}(a) \in U$. Since $J_{i}(a) \in U$ and $J_{i}(a) \supset J_{j}(b) \in U$ in the Boolean algebra $E(\mathbf{A})$ implies that $J_{j}(b) \in U, F$ is closed under modus ponens. It is easier to see that $F$ is closed by the other rules.

To prove that condition 1 implies condition 2 , let $F$ be an $\&$-filter, and let $U=F \cap E(\mathbf{A})$. From the Boolean axiom and the definition of $\&$-filter, $U$ is a filter on $E(\mathbf{A})$ and $F=\left\{a \in A: J_{n-1}(a) \in U\right\}$.

Lemma 4.6 Let $F=\left\{a \in A: J_{n-1}(a) \in U\right\}$ be a Boolean filter. Then $\theta(F)=$ $\left\{(a, b) \in A^{2}: J_{i}(a) \supset J_{i}(b) \in U, J_{i}(b) \supset J_{i}(a) \in U\right.$ for each $\left.i=0, \ldots, n-1\right\}$ is a Boolean congruence and verifies $\theta=\Omega_{A}(F)$.

Proof Clearly, if $\eta$ is the congruence on $E(\mathbf{A})$ defined by $U$, that is,

$$
\eta=\left\{(x, y) \in E(A)^{2}: x \supset y \in U, y \supset x \in U\right\}
$$

and $\bar{\eta}$ is the Boolean congruence given in Section 3 , then $\theta(F)=\bar{\eta}$.

By Theorem 1.6 of [3], to prove that $\theta=\Omega_{A}(F)$, it is enough to see that $\theta$ is elementarily definable in $\varsigma_{D}$. This means that there exists a first-order formula $X$ with parameters and without equality in $\S_{D}$ such that $a \theta b$ if and only if $\vDash_{\mathbf{A}, v(p / a, q / b)} X$. Recalling that the interpretation of $D$ is $F$, let $X$ be the formula

$$
D\left(\bigwedge_{i=0}^{n-1}\left(J_{i}(p) \supset J_{i}(q)\right) \wedge\left(J_{i}(q) \supset J_{i}(p)\right)\right)
$$

Then $\vDash_{\mathbf{A}, v(p / a, q / b)} \quad D\left(\bigwedge_{i=0}^{n-1}\left(J_{i}(p) \supset J_{i}(q)\right) \wedge\left(J_{i}(q) \supset J_{i}(p)\right)\right)$ if and only if $\bigwedge_{i=0}^{n-1}\left(J_{i}(a) \supset J_{i}(b)\right) \wedge\left(J_{i}(b) \supset J_{i}(a)\right)$ belong to $F$. Note that if

$$
c=\bigwedge_{i=0}^{n-1}\left(J_{i}(a) \supset J_{i}(b)\right) \wedge\left(J_{i}(b) \supset J_{i}(a)\right) \in E(A),
$$

then $c \in F$ if and only if $c \in U$, and therefore $a \theta b$ if and only if $\vDash_{\mathbf{A}, v(p / a, q / b)} X$.

Lemma 4.7 Let $\mathbf{A}$ be an algebra, $\mathbf{A} \in \mathcal{M}$, and let $\mathcal{D}$ be the family of 8 -filters on A. Let $\Theta$ be given by

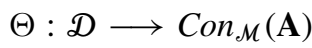

$$
\begin{aligned}
& F \longmapsto \theta(F)
\end{aligned}
$$

with $\theta(F)$ as in Lemma 4.6. Then the map $\Theta$ is an order isomorphism.

Proof Let $\mathbb{D}$ be the map: $\mathbb{D}: \operatorname{Con}_{\mathcal{M}}(\mathbf{A}) \longrightarrow \mathscr{D}, \bar{\eta} \longmapsto F$, where $F$ is the Boolean filter given by the filter $U \subseteq E(\mathbf{A})$ associated with $\eta$. Clearly, $\mathbb{D}=\Theta^{-1}$. Also, it is easy to see that $\Theta$ preserves the order: if $F \subseteq F^{\prime}$ then $\theta(F) \subseteq \theta\left(F^{\prime}\right)$.

From Lemmas 4.6 and 4.7 we can conclude the following.

Theorem 4.8 For any algebra $\mathbf{A} \in \mathcal{M}$, the Leibnitz operator $\Omega_{\mathbf{A}}$ is an isomorphism between the lattices of 8 -filters and $\mathcal{M}$-congruences of $\mathbf{A}$.

Theorem 4.3 follows from Lemmas 4.6 and 4.7 and from Theorem 4.4. 
Lemma 4.9 Let $\Delta$ be the system of formulas in two variables and let $E$ be the single

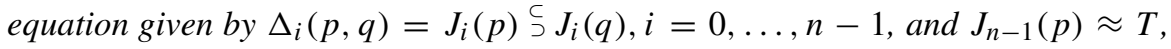
respectively. Then the following conditions hold for all $X_{1}, X_{2}, X_{3} \in \mathcal{F}$ :

1. $\vdash X_{1} \Delta X_{1}$,

2. $X_{1} \Delta X_{2} \vdash X_{2} \Delta X_{1}$,

3. $X_{1} \Delta X_{2}, X_{2} \Delta X_{3} \vdash X_{1} \Delta X_{3}$. For every primitive connective $*$ and all $X_{1}, \ldots, X_{k}, Y_{1}, \ldots, Y_{k} \in \mathcal{F}$, where $k$ is the rank of $*$, it holds that

4. $X_{1} \Delta Y_{1}, \ldots, X_{k} \Delta Y_{k} \vdash *\left(X_{1}, \ldots, X_{k}\right) \Delta *\left(Y_{1}, \ldots, Y_{k}\right)$, and for all $X \in \mathcal{F}$ it holds that

5. $X \vdash J_{n-1}(X) \Delta T$ and $J_{n-1}(X) \Delta T \vdash X$.

Proof The conditions (1) - (3) are immediate.

(4) The fact that $\Delta$ is a congruence relation on $\mathcal{F}$ is a direct consequence of the Boolean axioms, Axiom (Cn-G), and Axiom $\left(\mathrm{CJ}_{i}\right)$.

(5) First we observe that $J_{n-1}(X) \Delta T$ are the formulas

$$
J_{0}\left(J_{n-1}(X)\right) \stackrel{\complement}{丂} J_{0}(T), \ldots, J_{i}\left(J_{n-1}(X)\right) \subsetneq J_{i}(T), \ldots, J_{n-1}\left(J_{n-1}(X)\right) \stackrel{\complement}{丂} J_{n-1}(T) .
$$

From the Boolean and $\left(\mathrm{CJ}_{i}\right)$ axioms, it follows that to prove (5) it is sufficient to see that $X \vdash J_{n-1}(X) \stackrel{\complement}{\supset} T$ and $J_{n-1}(X) \stackrel{\complement}{\supset} T \vdash X$, which in turn is a consequence of the deduction rules and the Boolean axioms.

From Lemma 4.9 and from [3] (see Theorem 4.7) the following corollary is immediate.

Corollary 4.10 $\Delta$ and $E$ are systems of equivalence formulas and defining equations for \& and $\mathcal{M}$.

The following result was already proved in [2].

Corollary 4.11 (Completeness Theorem) $\quad$ For each $\Gamma \subset \mathcal{F}$ and each $X \in \mathcal{F}$, if $\Gamma \vDash X$, then $\Gamma \vdash X$.

Proof First we observe that if $v$ is a valuation on $\mathbf{A} \in \mathcal{M}, v(X)=1$ if and only if $v\left(J_{n-1}(X)\right)=1$. Also, we observe that from the corollary, it is enough to see that if $\Gamma \vDash X$, then $\left\{J_{n-1}(Y) \approx T: Y \in \Gamma\right\} \vDash J_{n-1}(X) \approx T$. Let $v$ be a valuation on $\mathbf{A}$ $\in \mathcal{M}$. If $v\left(J_{n-1}(Y)\right)=1$ for each $Y \in \Gamma$, then $v(Y)=1$ for each $Y \in \Gamma$, and from hypothesis $v(X)=1$. Therefore, $v\left(J_{n-1}(X)\right)=1$.

\section{Axiomiomatization with Truth Values Designated}

We note some facts in order to generalize the results in the case of more designated values. Now, let $D \subset M_{n}$ be such that $0 \notin D, 1 \in D$ and $D$ verifies the conditions, $\left(\mathrm{C}_{D \supset}\right)$ : there exists $d \in D, d \supset x \in D$, then $x \in D$, and $\left(\mathrm{C}_{D \vee}\right): x \vee y \in D$ if and only if $x \in D$ or $y \in D$. D is the set of designated truth values.

Consider the set of formulas and axioms as above. The rules of inference are modus ponens, $\mathrm{J}_{D}$-introduction $\left(\frac{X}{\nabla_{\alpha \in D} J_{\alpha}(X)}\right)$, and $\mathrm{J}_{D}$-elimination $\left(\frac{\bigvee_{\alpha \in D} J_{\alpha}(X)}{X}\right)$. Let $\varsigma_{D}$ be this propositional logic.

A formula $X$ is a tautology for $\mathcal{M}$ if $v\left(\bigvee_{\alpha \in D} J_{\alpha}(X)\right)=1$ for each valuation $v$ on $\mathbf{A}$, for any $\mathbf{A} \in \mathcal{M}$. Observe that from condition $\left(\mathrm{C}_{D \vee}\right), X$ is a tautology if 
and only if there exists $\alpha \in D$ such that $J_{\alpha}(X)=1$. It follows from $\left(\mathrm{C}_{D \vee}\right)$ that every derivable formula is a tautology. Now $F$ is a Boolean filter if and only if $F=\left\{a \in A: \bigvee_{\alpha \in D} J_{\alpha}(a) \in U\right\}$, where $U$ is a filter on $E(A) . \delta_{D}$ is algebraizable with equivalent algebraic semantic $\mathcal{M}$. Now the defining equation $\delta(p) \approx \epsilon(p)$ is the single equation $\bigvee_{\alpha \in D} J_{\alpha}(p) \approx T$.

\section{References}

[1] Abad, M., Estructuras Cíclica y Monádica de un Álgebra de Łukasiewicz n-valente, vol. 36 of Notas de Lógica Matemática, Universidad Nacional del Sur Instituto de Matemática, Bahía Blanca, 1988. MR 935965. 217

[2] Anshakov, O., and S. Rychkov, "On finite-valued propositional logical calculi," Notre Dame Journal of Formal Logic, vol. 36 (1995), pp. 606-29. Zbl 0852.03009. MR 96k:03059. 211,219, 223

[3] Blok, W. J., and D. Pigozzi, "Algebraizable logics," Memoirs of the American Mathematical Society, vol. 77 (1989), pp. 1-78. Zbl 0664.03042. MR 90d:03140. 211, 221, 222, 223

[4] Boicescu, V., A. Filipoiu, G. Georgescu, and S. Rudeanu, Eukasiewicz-Moisil Algebras, vol. 49 of Annals of Discrete Mathematics, North-Holland Publishing Co., Amsterdam, 1991. Zbl 0726.06007. MR 92k:03031. 216, 220

[5] Burris, S., and H. P. Sankappanavar, A Course in Universal Algebra, vol. 78 of Graduate Texts in Mathematics, Springer-Verlag, New York, 1981. Zbl 0478.08001. MR 83k:08001. 219

[6] Cignoli, R., and M. S. de Gallego, "The lattice structure of some Łukasiewicz algebras," Algebra Universalis, vol. 13 (1981), pp. 315-28. Zbl 0495.03045. MR 83d:06013. 216, 217

[7] Pigozzi, D., "Finite basis theorems for relatively congruence-distributive quasivarieties," Transactions of the American Mathematical Society, vol. 310 (1988), pp. 499-533. Zbl 0706.08009. MR 90b:08014. 218

[8] Rosser, J. B., and A. R. Turquette, Many-valued Logics, Studies in Logic and the Foundations of Mathematics, North-Holland Publishing Co., Amsterdam, 1951. Zbl 0047.01503. MR 14,526a. 211

\section{Acknowledgments}

I would like to express gratitude to Dr. R. Cignoli for the help he gave during the preparation of this paper. Also I wish to thank the referee for his/her helpful comments to improve its presentation.

Departamento de Matemática del Ciclo Básico Común

Universidad de Buenos Aires

ARGENTINA

oambas@dm.uba.ar 